\title{
An Traffic Subarea Partition Algorithm based on State recognition
}

\author{
Liangzhi Zhang ${ }^{1, a^{*}}$, Peide $\mathrm{Li}^{2, \mathrm{~b}}$ \\ ${ }^{1}$ College of Traffic and Logistic Engineer, Shandong Jiaotong University, Jinan, Shandong \\ ${ }^{2}$ Shandong Jinan Power Equipment Factory Co. LTD., Jinan, Shandong

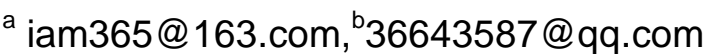 \\ * please mark the corresponding author with an asterisk
}

Keywords: State recognition, Subarea partition, Traffic control

Abstract. Traffic State subarea partition problem has been a hot topic. A sub-area partition algorithm is proposed in this paper based on state connectivity matrix with flow variance in intersections within the road network. The principle of subarea partition is posed in the paper, and a dynamic algorithm is designed based on the principle. An example demonstrates the effectiveness of the algorithm.

\section{Introduction}

As a research focus and challenge in city traffic, area control is different from single intersection signal control in that the former directs at user equilibrium. That is, it aims at the optimal signal control strategy in all intersections within the road network with user equilibrium assignment. This is a control optimization problem which is signaled as Equilibrium Area Control. A variety of intelligence algorithms such as Support Vector Machine、Decentralized coordination、Bidirectional particle swarm etc are applied to area control. Abdulaal firstly suggested HJ with determined continuous decision variables and feasible region, which is more efficient for small scale road network constrained by computation volume [1]. Feizhou Zhang designed traffic coordination control system based on multi Agent and established an intelligent traffic control system framework. But the solution algorithm design is a hard problem [2]. Jianmin Xu carried out research from the perspective of traffic signal phase matching with consideration of area road network [3]. Some studies explored algorithm incorporating state estimation into model predictive control which was applied to road network traffic control $[4,5]$. In the cited algorithms area traffic state is not fully depicted and control strategy is greatly affected by traffic state. Hence weight coefficient of traffic state is introduced to algorithm model to impose more effective control over traffic in this paper.

In this paper, based on an analysis of road state, state index is defined and a sub-area partition algorithm based on pass rate is put forward. Simultaneously, a gradient projection solution algorithm is designed based on projection algorithm according to the variational inequality formula in area control model, which brings new ideology to urban road network area control.

\section{Area traffic state analysis}

$\mathrm{G}(\mathrm{N}, \mathrm{L})$ is area road network under control of the same one signal where nodes set $\mathrm{N}$, links set $\mathrm{L}$ respectively are $\mathrm{N}=\{1,2 \ldots \mathrm{m}\}$ and $\mathrm{L}=\left\{l_{i j} \mid l_{i j}=<\mathrm{i}, \mathrm{j}>, \mathrm{i}, \mathrm{j} \in \mathrm{N}, \mathrm{i}, \mathrm{j}=1,2 \ldots \mathrm{m}\right\}$;

$q_{r s}$ is overall traffic demand of OD pair r-s ;

$q_{r s}^{u}$ is the traffic demand of path u connecting r-s ;

$q_{i j}^{x}$ is traffic flow of link $l_{i j}$ in all directions where $\mathrm{x}=1,2,3$ denotes flow of left, straight and right respectively ;

$q_{i j}$ is traffic flow from node $\mathrm{i}$ to node $\mathrm{j}, q_{i j}=\sum_{x} q_{i j}^{x}$; 
$q_{i j}^{r s}$ is flow of link $\mathrm{l}_{\mathrm{ij}}$ connecting pair r-s ;

$\delta_{i j}^{r s u}$ is correlation coefficient of OD pair r-s and link $1_{\mathrm{ij}}, \quad \delta_{i j}^{r s u}=0$.If link $1_{\mathrm{ij}}$ is on the path $\mathrm{u}$ connecting pair r-s, we have $\delta_{i j}^{r s u}=1$, otherwise $\delta_{i j}^{r s u}=0$;

$t_{i j}^{x}$ is mean travel resistance of link $l_{i j}$ in $\mathrm{x}$ direction( $\mathrm{X}$ signal group) ;

$r_{i j}^{x}$ is road traffic state coefficient denoting the mean travel time per unit length of link $l_{i j}$ in $\mathrm{x}$ direction , $r_{i j}^{x}=t_{i j}^{x} / d_{i j}$ where $d_{i j}$ is length of $l_{i j}$;

Adjacent node matrix $\mathrm{A}=\left\{a_{i j}\right\}$, where

$$
a_{i j}=\left\{\begin{array}{c}
1 \quad i, j \in N \text { and }<i, j>\in L \\
0 \quad i, j \notin N \text { or }<i, j>\notin L
\end{array}\right.
$$

Congestion value $\lambda$ is the reciprocal of average travel speed, that is $\lambda=1 / \mathrm{v}$.

According to China road traffic management evaluation indicators, in this paper, $\lambda_{1}=$ 0.2 (equivalent to $5 \mathrm{~km} / \mathrm{h}$ ) and $\lambda_{2}=0.1$ (equivalent to $10 \mathrm{~km} / \mathrm{h}$ ) represent cut-off value of serious congestion; $\lambda_{3}=0.066$ (equivalent to $15 \mathrm{~km} / \mathrm{h}$ ) and $\lambda_{4}=0.05$ (equivalent to $20 \mathrm{~km} / \mathrm{h}$ ) are cut-off value of moderate congestion; $\lambda_{5}=0.04$ (equivalent to $25 \mathrm{~km} / \mathrm{h}$ ) and $\lambda_{6}=0.033$ (equivalent to $30 \mathrm{~km} / \mathrm{h}$ ) are of slight congestion ${ }^{[14]}$.

Define traffic state connectivity matrix $C\left(\lambda_{\tau}\right)$ to be a matrix that reflects whether the given link is accessible with the threshold $\lambda_{\tau}$ specified in the above $C\left(\lambda_{\tau}\right)=\left\{c_{i j}\left(\lambda_{\tau}\right)\right\}$, where matrix element value is

$$
c_{i j}\left(\lambda_{\tau}\right)=\left\{\begin{array}{cc}
1 & r_{i j}^{x}<\lambda_{\tau} \text { and } a_{i j}=1 \\
0 & r_{i j}^{x} \geq \lambda_{\tau} \text { or } a_{i j}=0
\end{array}\right.
$$

\section{Traffic control sub-area partition algorithm}

Junction nodes are traffic bottleneck directly affecting the road state in spite of their small geographical area and the spots where control strategies are mainly implemented. Thus a traffic control area is partitioned according to its node state. A control area is partitioned in a dynamic way with traffic states varying continuously in terms of time and space. Traffic optimization and control guarantees the most sufficient performance of traffic system with equilibrium distribution of vehicles on all junctions and links whereby local congestion is avoided. Therefore, congested junctions group is the focus of traffic control. A congested region and neighboring dispersed junctions are put into the same area so that coordinated control can be implemented in the junctions within the control area and congested vehicles can be diverged, whereby coordinated timing of signals on all junctions is realized. follows,

Based on the above, the algorithm of traffic control sub-area partition at specific time is given as

Step 1: With node set $N^{\prime}=\mathrm{N}$ given, establish $\mathrm{N}$ node sets $\mathrm{Ns}=\Phi, \mathrm{s}=1 \ldots \mathrm{m}$, calculate pass degree of node $\mathrm{j}(\mathrm{j}=1 \ldots \mathrm{m}, \mathrm{j} \in \mathrm{N}) p_{j}, p_{j}=\sum_{\lambda_{\mathrm{r}}} \sum_{i} \lambda_{\mathrm{\tau}} c_{i j}\left(\lambda_{\mathrm{\iota}}\right) \quad$, assume $\mathrm{j}=1, \mathrm{~s}=1$;

Step 2: If $\mathrm{j}=\mathrm{m}$, switch to Step 6;

Step 3: If $\mathrm{j} \notin N^{\prime}$ and $\mathrm{j} \in \mathrm{Ns}, \exists \mathrm{s}$, we derive $\mathrm{j}=\mathrm{j}+1$,switch to Step 2;

Step 4: For the connectivity state $c_{i j}\left(\lambda_{\tau}\right)$ of all the links $1_{\mathrm{ij}} \in \mathrm{L}\left(\mathrm{i}=1 \ldots \mathrm{m}, \mathrm{j} \in N^{\prime}\right)$, if $c_{i j}\left(\lambda_{\tau}\right)=0$ with the given value of $\lambda_{\tau}\left(\lambda_{\tau}=0.2\right.$ or 0.1$)$, carry out the following procedures:

(1) If $\mathrm{i} \in \mathrm{Np}$, derive $\mathrm{N}=\mathrm{N}-\{\mathrm{j}\}, \mathrm{Np}=\mathrm{Np} U\{\mathrm{j}\}$; otherwise, $N^{\prime}=N^{\prime}-\{\mathrm{j}\}, \mathrm{Ns}=\mathrm{Ns} \cup\{\mathrm{j}\}, \mathrm{s}=\mathrm{s}+1$;

(2) Depth-First traverse set $\mathrm{N}_{\mathrm{p}}\left(\mathrm{orN}_{\mathrm{s}}\right)$ to which $\mathrm{j}$ belongs , for each node $\mathrm{x}$, assume $\mathrm{j}=\mathrm{x}$, step 4 then is applied; All the links are processed. Then switch to Step 5; 
Step 5: $\mathrm{j}=\mathrm{j}+1$, switch to Step 2 ;

Step 6: Carry out procedures in all the non-empty sets $\mathrm{N}_{\mathrm{q}}, \mathrm{q}=1$...Q as follows:

For all the nodes $\mathrm{i}, \mathrm{i} \in \mathrm{N}_{\mathrm{q}}$, if node $\mathrm{j}$ satisfies $\mathrm{l}_{\mathrm{ij}} \in \mathrm{L}$, and $\mathrm{j} \in \mathrm{N}$, derive $N^{\prime}=N^{\prime}-\{\mathrm{j}\}$ and $\mathrm{N}_{\mathrm{q}}=\mathrm{N}_{\mathrm{q}} \mathrm{U}\{\mathrm{j}\}$;

Step 7: Arrange the remaining n nodes of $N^{\prime}$ in pass-degree order from low to high ;

Step 8: procedures are implemented in node i of $N^{\prime}$ in pass-degree order from low to high :

(1) $N^{\prime}=N^{\prime}-\{\mathrm{i}\}, \mathrm{s}=\mathrm{s}+1, \mathrm{Ns}=\mathrm{Ns} \cup\{\mathrm{i}\}$

(2) For node $j$ satisfying $l_{i j} \in L$, if $j \in N$, we have :

(a) $N^{\prime}=N^{\prime}-\{\mathrm{j}\}$, Ns=NsU $\{\mathrm{j}\}$;

(b) For node k satisfying $\mathrm{l}_{\mathrm{jk}} \in \mathrm{L}$, if $\mathrm{k} \in \mathrm{N}$ and $p_{k} \leq p_{j}$, we have $\mathrm{N}=\mathrm{N}-\{\mathrm{k}\}, \mathrm{Ns}=\mathrm{Ns} \mathrm{U}\{\mathrm{k}\}$;

\section{Numerical examples}

To test the above model and algorithm, optimization calculation is carried out in a real road network. The control algorithm program is written with MATLAB and the calculation result is input to the simulation tool, VISSIM.

Nguyen-Dupuis is brought as a research road network as shown in Fig. 1.This network consists of 12 junctions, 19 links and 4 OD pairs. Table 1 displays lanes number in each link, free travel time and link capacity. Junctions 1、2、3、4 are traffic attraction points containing 8 OD pairs. Traffic demand among each OD pair subjects to logarithmic normal distribution with average $q_{r s, a v}$ v variance $\varepsilon_{r s}$. Traffic demand in flat periods, peak hours and transition periods is displayed in Table 2. Each period is split into 3 moments and random variables subjecting to distribution are derived in each moment.

In this road network, standard four-phase control is implemented in cross intersections and three-phase control in T-shaped intersections. The link length is set at the same proportion as in the figure.

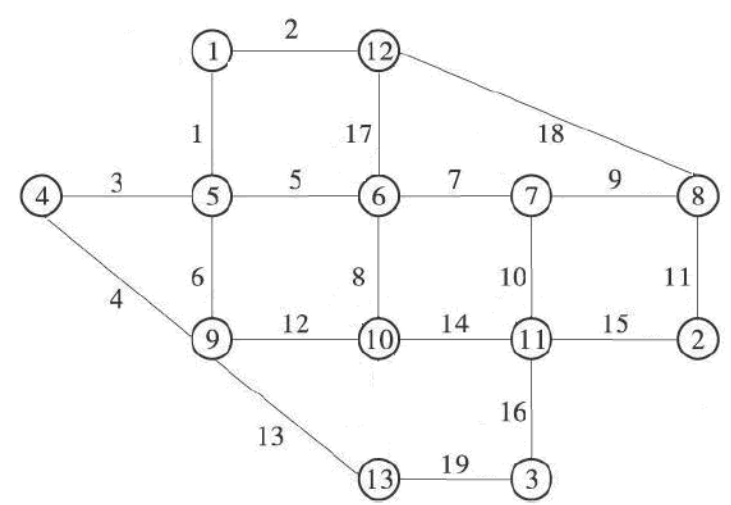

Fig.1 Nguyen-Dupuis Road Network sample

Based on the above assumptions, operate program and obtain control scheme as shown in table 2 . It is then simulated in VISSIM until it reaches a stable state. Results of this algorithm and TTANSYT are compared in Table 3. It can be seen that in both control schemes total delay time and total number of vehicles are almost the same within flat periods. Improvements in this proposed model are rather modest with total delay time only $2.2 \%$ less. But total del ay time is decreased by $7.5 \%$ and total traffic flow is increased by $5.3 \%$ within peak periods, which means substantial improvement in this model. It is demonstrated that TRANSYT is only viable for low-saturated traffic states. 
Table 1 Nguyen-Dupuis parameters in road network links

\begin{tabular}{|c|c|c|c|}
\hline Link number a & $t_{a 0}(\mathrm{Min})$ & $\begin{array}{c}\text { Number of } \\
\text { lane }\end{array}$ & $\begin{array}{c}C_{a}(\text { vehicle } \\
\text { number } / \mathrm{h})\end{array}$ \\
\hline 1 & 5 & 3 & 3200 \\
\hline 2 & 6 & 2 & 2400 \\
\hline 3 & 8 & 2 & 2500 \\
\hline 4 & 12 & 2 & 2200 \\
\hline 5 & 6 & 3 & 3100 \\
\hline 6 & 7 & 3 & 3200 \\
\hline 7 & 5 & 2 & 2400 \\
\hline 8 & 7 & 3 & 3300 \\
\hline 9 & 9 & 2 & 2500 \\
\hline 10 & 7 & 2 & 2300 \\
\hline 11 & 7 & 2 & 2200 \\
\hline 12 & 6 & 2 & 2300 \\
\hline 13 & 9 & 3 & 3200 \\
\hline 14 & 5 & 3 & 3500 \\
\hline 15 & 9 & 3 & 3300 \\
\hline 16 & 5 & 2 & 2500 \\
\hline 17 & 5 & 2 & 2300 \\
\hline 18 & 15 & 2 & 2400 \\
\hline 19 & 5 & 3 & 3300 \\
\hline
\end{tabular}

Table 2 Logarithmic distribution parameters of OD pairs traffic demand

\begin{tabular}{|c|c|c|c|c|}
\hline \multirow{2}{*}{ OD pair } & \multicolumn{2}{|c|}{ Flat period } & \multicolumn{2}{c|}{ Peak hours } \\
\cline { 2 - 5 } & average $q_{r s, a v}$ & variance $\varepsilon_{r s}$ & Average $q_{r s, a v}$ & variance $\varepsilon_{r s}$ \\
\hline 1-2、 & & & & \\
$4-3 、$ & 6.4 & 0.1 & 7.35 & 0.12 \\
$3-4$ & & & & \\
\hline $1-3 、$ & & & & \\
$3-1$ & 6.2 & 0.1 & 7.2 & 0.12 \\
$4-2 、$ & & & & \\
$2-4$ & & & & \\
\hline
\end{tabular}

Control sub-area partition determines control effect. Fig 2 displays control sub-area partition for flat and peak hours. It can be seen that 2 or 3 junctions compose one sub-area or even one single junction is treated as one sub-area when traffic is smooth with small flow and influence between junctions is minor. In peak hours traffic is over-saturated and congested junctions increase and smooth junctions coordinate with neighboring congested ones. In this case a single control sub-area enlarges with decreasing number. 


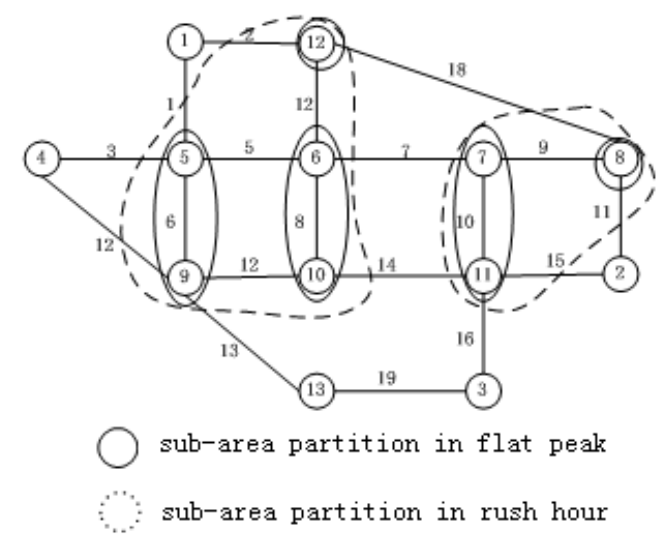

Fig.2 Traffic control sub-area partition under different traffic states

\section{Summary}

Alleviation of urban roads congestion at peak periods is an urgent problem for both traffic managers and researchers. Congestion is mainly caused by junction delay which varies greatly in different junctions. Equilibrium assignment in control sub-area junctions is fully guaranteed to improve traffic in this paper with consideration of vehicle delay coefficient of congested junctions.

Preliminary analysis and evaluation of this proposed model is conducted in this paper. And more thorough experiments will be done on this model in more various traffic demands and larger road networks. In addition, higher calculation efficiency and seeking superior algorithm to this model is the further work to be tackled.

\section{Acknowledgements}

This work was financially supported by the Ministry of Transport Science and Technology Projects (2012-319-817-230), Natural Science Foundation of Shandong Province (ZR2012FL02) and Shandong Higher Education Science and Technology Projects (J10LG52).

\section{References}

[1] M. Abdulaal, L.J. LeBlanc, Continuous equilibrium network design models, Transportation Research Part B,1979, pp.19-32.

[2] Z. Feizhou , C. Xuejun , S. Min , Design for urban traffic integrated control system based on multi-agent, Acta Scientiarum Naturalium Universitatis Pekinensis, 2008, pp.289-292.

[3] X. Jianmin, S. Yanfang, L. kai , Area Coordinated Control of Traffic Signals Based on Bi-Level Programming Model, Journal of South China University of Technology, 2011,pp.95-101.

[4] Z. Bo, J. Zhicai, L. Xuxun, Stochastic user equilibrium model based on cumulative prospect theory, journal of southwest jiaotong university, 2011, pp.868-874.

[5] S. Qingying, G. Bao , J. Ling, Modified conjugate gradient projection method for nonliner constrained optimization, acta mathemacticae applicate sinica, 2010,pp.640-651. 